\title{
What are the best payment models to support population health?
}

\section{Opinion}

Hospitals and health systems in the US have been facing mounting pressure to improve patient outcomes while reducing costs. They have, as a result, been focusing more on population health management and adopting other initiatives such as patient-centered medical homes that encourage continuity between hospitals, physicians, specialists, skilled nursing facilities and other care providers. The current system, however, is deeply flawed as far as physician reimbursement is concerned. Primary care physicians only get reimbursed primarily on the basis of volume of patient visits and little else. Physicians are not reimbursed for services such as phone or email consultations with patients, both of which help monitor patients' health and cut costs and improve outcomes. There is a recognition in the health sector that a fee-for-service system that pays for volumes rather than quality of care is not the best approach.

\section{The new population health payment system}

Progress has been made; a transition from fee-for-service to a payment system gives hospitals and health systems an incentive to jump onboard. Providers involved in ACO are adopting a pay-forperformance model that reimburses physicians on a number of metrics including patient safety and outcomes, care quality and cost of care. In 2014, CMS held back $1.25 \%$ of Medicare reimbursements at hospitals under the new Medicare inpatient prospective payment system. The cost saving, which is estimated to be $\$ 1.1$ billion, will be dispersed to hospitals based on their performance on various healthcare quality measures. ${ }^{1}$ Health insurers are also joining the push for performancebased systems. United Healthcare, a leading health insurance provider, plans to double its value-based contracts with providers.

\section{State payment reform to reduce hospital re-admissions}

The need for payment reform has partly been triggered by the inability to manage the burgeoning healthcare costs, the need to deliver quality care and to reduce unnecessary re-admissions. The current reimbursement models in many States do not pay for things that have the potential to reduce hospital re-admissions, such as care transition coaches who help patients who return home after hospitalization, or nurse care managers who visit chronically sick patients to educate them and provide self-management support, or other methods such as using tele-monitoring to identify problems long before they occur. that:

Several states are exploring various payment reform methodologies

i. Avoid paying providers for patient re-admissions.

ii. Pay providers more to encourage them to implement programs that have the potential to reduce re-admissions.

iii. Give bonuses to providers or levy penalties based on re-admission rates.

iv. Make comprehensive care payments to providers for all patients care needs regardless of the number of hospitalizations or readmissions they require.
Volume 2 Issue 4 - 2015

\author{
Miguel Mclnnis \\ Mclnnis \& Associates Consulting LLC, USA
}

Correspondence: Miguel Mclnnis LLC, Mclnnis \& Associates Consulting LLC, 700 I2th St. NW, Suite 700 Washington, DC 20005, USA, Tel I8662279457,

Email info@mcinnisassociates.com

Received: May 22, 2015 | Published: July 02, 2015

v. Pay for healthcare with a limited warranty from the provider, so that the provider does not charge for re-admission that meet certain set criteria.

The ability to titrate the right payment model will be key. States that adopt an overly strict stance towards re-admissions, however, can have several undesirable consequences. The providers can legitimately decline to treat re-admitted patients if the payer refuses to pay for readmissions. In other cases, the patient might be readmitted to another hospital other than the one where initial care was provided, or treated by other physicians. In extreme cases, hospitals might simply refuse to admit patients if they feel the patients have high chances of readmissions. Recognizing that not all re-admissions are preventable. It's also worth remembering that a highly successful pay-for-performance (P4P) program that rewards providers who successfully reduce readmissions can end up hurting the hospitals revenue in a big way and thus provide a strong disincentive for providers to enforce it. In light of these facts, I believe that many states will follow Medicare's lead in developing similar payment reform models that provide a workaround while offering a balanced solution.

Medicare has proposed the following strategy to help reduce readmissions:

i. All DRG payments were reduced by up to $2 \%$ in the current year and $3 \%$ in 2015.

ii. The actual reductions are calculated using excess risk-adjusted re-admissions for conditions such as heart failure, heart attacks and pneumonia.

iii. More conditions will be added to the list in 2015 . $^{2}$

In theory, the Medicare strategy works better than most existing $\mathrm{P} 4 \mathrm{P}$ programs because it magnifies the penalty for high re-admission rates for certain targeted conditions and the program continues to pay nearly the same for other re-admissions whenever they occur. The biggest drawback of the system is that it would disproportionately penalize (urban and rural) hospitals located in high-risk underserved service areas with patients who possess the target conditions while offering no incentive to reduce re-admissions for patients with other conditions. Developing a comprehensive payment reform model is quite complex given the many moving parts that have to be taken care 
of. Even though a perfect system is yet to be developed, many states are "tinkering" with a variety of payment models.

\section{The state of Maryland's health reform model}

Early on, the State of Maryland realized that the disadvantages of a fragmented system of care were:

i. There are few incentives for improved quality of services or cost control

ii. Poor resource allocation and high administrative costs

iii. Hospitals have the most market power, which naturally keeps medical costs high

The state established the Health Services Cost Review Commission (HSCRC) to govern hospital rates throughout the state. Consequently, Maryland is the only state in the US that sets uniform prices for all insurers. The state also became the first to establish a first-of-itskind hospital regulatory scheme aimed at reducing hospital spending beginning early 2014 .

\section{HSRC and all-payer system}

The Health Services Cost Review Commission (HSRC) statute was enacted in 1971. The commission began setting hospital rates in 1974 with its authority at the time limited to rates that hospitals charged non-governmental purchasers of healthcare services. In 1977, Maryland alongside four other states including New York, Massachusetts, Connecticut and New Jersey was granted a waiver by the federal government exempting it from national Medicaid and Medicare reimbursement principles. Since then, payers pay Maryland hospitals according to rates established by the HSCR using the famous All-Payer system. The commission ensures that:

i. Total costs of services offered by hospitals are reasonable.

ii. Hospital rates are set equitably among all hospital services purchasers.

iii. Aggregate hospital revenues are reasonably to its aggregate costs. $^{3}$

The other four states abandoned the practice in the 1980s as managed care organizations become increasingly popular. The thinking back then was that managed care companies would naturally be interested in keeping their costs down in a bid to remain competitive and thus help to keep hospital spending down. This has, however, not worked as expected.

\section{All-payer system upgraded}

The state of Maryland in conjunction with the Centers for Medicare and Medicaid Services (CMS) have partnered to modernize the state's unique all-payer system for hospitals services that will be used to improve patients' health outcomes while reducing healthcare costs. The new initiative will gradually update Maryland's 36-year old Medicare waiver and allow the state to adopt new policies that will lower per capital health expenditure and improve patients' health outcomes as is encouraged by the Affordable Care Act.
The upgrades are scheduled to be done over a 5-year timeline as follows:

i. Hospital Global Models to be adopted during the current fiscal year (fiscal 2014).

ii. Population-based model scheduled to be implemented from FY 2015- FY 2017.

iii. Preparation for Phase 2 scheduled for implementation between FY 2016-FY 2019. ${ }^{4}$

\section{What do the changes mean?}

The proposed models represent the most significant changes in the mode of healthcare services payment in 40 years. Focus will shift to attaining the desired volumes and reducing utilization and consequently control the revenue budget. The increased efficiency will create new opportunities for better population health and improved care. Other key changes under the hospital global model (to be implemented from 2015-2017) include:

i. Total hospital per capita revenue growth for Maryland residents under the all-payer model will be tied to the state's long-term economic growth, which for the next 3 years is estimated at $3.58 \%$.

ii. The Medicare payment savings for Maryland residents will be comparable to the dynamic national trend.

iii. Align payments with new ways for organizing care.

iv. Evolve value payments around health outcomes and efficiency and not volumes or readmissions is currently the case.

The overall goal of the new payment models is to control the overall costs of healthcare while improving patient outcomes. Even though the model will at first be implemented in the state of Maryland, it could soon become the gold standard for other states to adopt, especially if it proves to be successful.

\section{Acknowledgements}

None.

\section{Conflict of interest}

The author declares no conflict of interest.

\section{References}

1. Albright EJ. CMS Announces New Medicare Reimbursement Rates for 2014. USA: insideARM. Com; 2013.

2. Center for Medicare and Medicaid Services. Readmissions Reduction Program. USA: CMS.gov; 2014.

3. Welcome to Health Services Cost Review Commission. USA: The Maryland Health Services Cost Review Commission; 2014.

4. All Payer Hospital System Modernization Payment Models Workgroup Meeting Presentation. USA: The Maryland Health Services Cost Review Commission; 2014. 